\title{
The nucleoporin Nup153 regulates embryonic stem cell pluripotency through gene silencing
}

\author{
Filipe V. Jacinto, Chris Benner, and Martin W. Hetzer \\ Molecular and Cell Biology Laboratory, Salk Institute for Biological Studies, La Jolla, 92037 California, USA
}

Nucleoporins (Nups) are a family of proteins best known as the constituent building blocks of nuclear pore complexes (NPCs), membrane-embedded channels that mediate nuclear transport across the nuclear envelope. Recent evidence suggests that several Nups have additional roles in controlling the activation and silencing of developmental genes; however, the mechanistic details of these functions remain poorly understood. Here, we show that depletion of Nup153 in mouse embryonic stem cells (mESCs) causes the derepression of developmental genes and induction of early differentiation. This loss of stem cell identity is not associated with defects in the nuclear import of key pluripotency factors. Rather, Nup153 binds around the transcriptional start site (TSS) of developmental genes and mediates the recruitment of the polycomb-repressive complex 1 (PRC1) to a subset of its target loci. Our results demonstrate a chromatin-associated role of Nup153 in maintaining stem cell pluripotency by functioning in mammalian epigenetic gene silencing.

[Keywords: nucleoporins; transcription; gene silencing; stem cell pluripotency; polycomb silencing]

Supplemental material is available for this article.

Received February 23, 2015; revised version accepted May 27, 2015.

Nuclear pore complexes (NPCs) are large nuclear envelope (NE)-embedded protein assemblies that are composed of multiple copies of $\sim 30$ different nucleoporins (Nups), creating a selective transport channel between the nucleus and the cytoplasm (D'Angelo and Hetzer 2008). Nups can be functionally separated into at least two categories: (1) the scaffold Nups, which consist of the Nup107/160 and Nup93/205 complexes that together comprise almost $50 \%$ of all Nups and form the stable core ring-like structure of the NPC (D'Angelo and Hetzer 2008; Toyama et al. 2013), and (2) the peripheral Nups, which establish the permeability barrier and mediate the translocation of cargo through the NPC via receptor-mediated processes (Wente and Rout 2010).

In addition to their roles as constituents of the NPC, Nups have emerged as potential regulators of chromatin organization and transcription (Van de Vosse et al. 2011; Pascual-Garcia and Capelson 2014). For example, studies in Saccharomyces cerevisae, Drosophila melanogaster, and mammalian systems have demonstrated that a subset of peripheral Nups, including Nup98 and Nup153, is able to physically interact with specific genomic loci and can regulate the transcriptional status of its target genes (Casolari et al. 2004; Schmid et al. 2006; Capelson et al. 2010; Kalverda et al. 2010; Vaquerizas et al. 2010; Liang

Corresponding author: hetzer@salk.edu

Article published online ahead of print. Article and publication date are online at http://www.genesdev.org/cgi/doi/10.1101/gad.260919.115. et al. 2013; Light et al. 2013; Van de Vosse et al. 2013). Importantly, in Drosophila these Nup-chromatin interactions were commonly found to occur in the nucleoplasm, away from the NE-embedded NPCs, suggesting that Nups may retain the ability to regulate gene activity even when not associated with NPCs. Furthermore, these Nup-gene contacts occur predominantly at developmentally regulated genes undergoing transcriptional induction $\mathrm{Ca}$ pelson et al. 2010; Kalverda et al. 2010). These studies uncovered functions for specific Nups that are not linked to mediating cargo translocation across the NPC channels. Instead, they suggest that Nups play a direct role in controlling developmental transcriptional programs. However, the role of the Nup-gene contacts during mammalian development remains obscure, and the molecular details of how Nups can participate in gene-activating and gene silencing processes is still a major unsolved question.

The observed role of Nups in developmental gene regulation is supported by reports of several NPC components exhibiting tissue-specific expression (Lupu et al. 2008; D'Angelo et al. 2012) and tissue-specific disease phenotypes (Zhang et al. 2008). Interestingly, peripheral Nups have also been shown to exhibit low residence times at

(C) 2015 Jacinto et al. This article is distributed exclusively by Cold Spring Harbor Laboratory Press for the first six months after the full-issue publication date (see http://genesdev.cshlp.org/site/misc/terms.xhtml). After six months, it is available under a Creative Commons License (Attribution-NonCommercial 4.0 International), as described at http:// creativecommons.org/licenses/by-nc/4.0/. 
the NPC, with a highly mobile, soluble pool being present inside the nucleoplasm (Rabut et al. 2004; Van de Vosse et al. 2011; Pascual-Garcia and Capelson 2014). Moreover, several mobile Nups, including Nup153, Nup98, and Nup50, are sensitive to transcriptional inhibition in mammalian cells, further implicating a link between Nups and transcriptional regulation (Griffis et al. 2004; Buchwalter et al. 2014). Recent genome-wide studies in several model organisms have demonstrated the importance of Nup153 in active transcription (Casolari et al. 2004; Vaquerizas et al. 2010). Nup153 has also been found to participate in X-chromosome transcriptional hyperactivation in dosage compensation of $D$. melanogaster (Mendjan et al. 2006). Altogether, Nup153 seems to be required for the establishment of active chromatin domains in flies. This raises the interesting question of whether Nup153 might play a function in developmental gene regulation in mammals.

Here, we show that mouse embryonic stem cells (mESCs) require Nup153 to maintain their pluripotency by mediating the recruitment of the polycomb-repressive complex 1 (PRC1) to the transcription start site (TSS) of several lineage-specific genes. Importantly, this function of Nup153 is not linked to the import of PRC1 components. Instead, Nup153 has the ability to bind to specific developmentally regulated genes and maintain them in a repressed state. Altogether, our study reveals a transport-independent role of Nup153 in PRC1-mediated gene silencing in mESCs, adding to the functional diversity of Nups in transcription control.

\section{Results}

\section{Nup153 knockdown causes differentiation of mESCs}

To study the potential role of Nup153 in stem cell function and differentiation, we constitutively expressed two different lentiviral shRNA vectors against Nup153 in mouse mESCs. Efficient knockdown of Nup153 was achieved with both constructs as verified by Western blotting analysis (Fig. 1A) and quantitative RT-PCR (qRT-PCR) (Supplemental Fig. S1A). Nup153 depletion resulted in a significant decrease of alkaline phosphatase (AP) staining (Fig. 1B) and in the formation of flat and morphologically distinct colonies indicative of early differentiation (Fig. 1C). We did not detect the cleavage of poly(ADP-ribose) polymerase (Parp1) in either of the two Nup153-depleted mESCs, suggesting that the observed loss of AP stem cell colonies was not the result of apoptosis (Fig. 1A; Supplemental Fig. S1E). Importantly, the phenotype was efficiently rescued by the expression of an shRNA-resistant mouse Flag-mCherry-Nup153, which properly localized
A

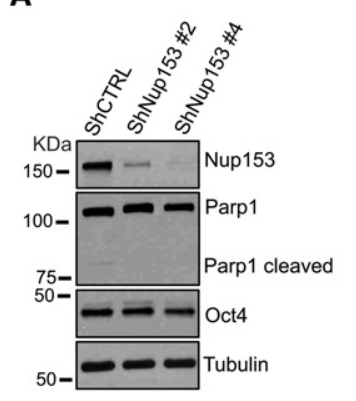

D

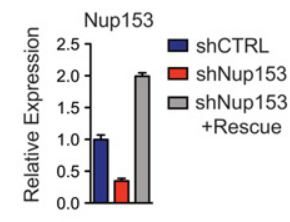

$\mathbf{F}$

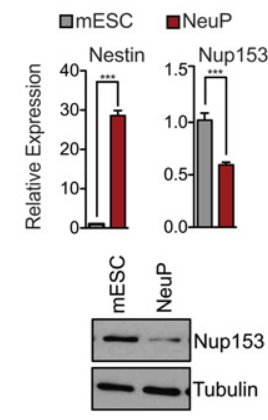

B

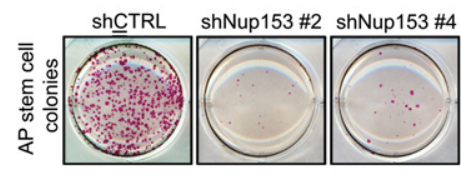

C

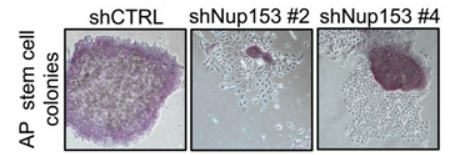

E

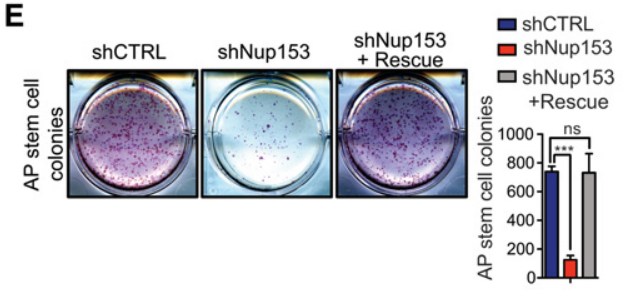

G

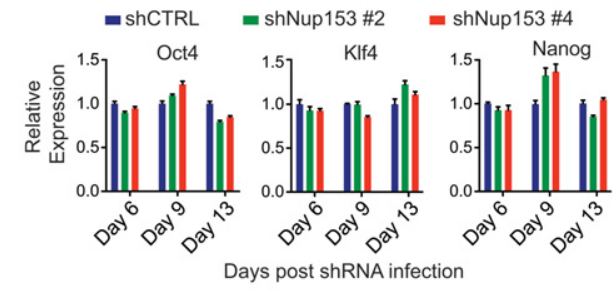

Figure 1. Nup153 knockdown induces the differentiation of mESCs that is not caused by the down-regulation of markers of the undifferentiated state. $(A)$ Western blot analysis for Nup153, Parp1, and Oct4 in whole-cell extracts from mESCs after $6 \mathrm{~d}$ of Nup153 knockdown. Tubulin was used as the loading control. (B) AP staining of control and stable Nup153depleted mESC colonies. The total number of positive AP stem cell colonies between the control and Nup153-deficient mESCs is quantified in the right graph. Data are represented as mean $\pm \mathrm{SD} ; n=3$. $P$-values were obtained from Student's $t$-test; $\left(^{* * *}\right) P<$ 0.001. (C) Representative phase-contrast microscopy images of control and Nup153 knockdown AP stem cell colonies. Original magnification, 100×. (D) Relative mRNA levels of Nup153 in control, knockdown, and rescued mESCs measured by qRT-PCR. The relative expression levels were normalized to actin and are expressed as fold change relative to the control (shCTRL). (E) AP staining (left panel) and total number of positive AP stem cell colonies (right graph) in control, Nup153 knockdown, and shRNAresistant Flag-mCherry-Nup153-expressing mESCs. (F) Nup153 expression analysis by qRT-PCR and Western blot upon the differentiation of mESCs into NeuP cells. Tubulin was used as the loading control. $(G)$ qRT-PCR analysis of the pluripotency factors Oct4, Nanog, and Klf4 after 6, 9, and $13 \mathrm{~d}$ of Nup153 knockdown. The relative expression levels were normalized to actin and are expressed as fold change to the respective control. The mean \pm SD from three independent experiments is shown. 
to the NE, indicating the functional integrity of this recombinant protein (Fig. 1E; Supplemental Fig. S1B,C). Analysis by qRT-PCR and Western blot revealed that the expression of the tagged Nup153 was $\sim 1.5$-fold to twofold higher than the endogenous Nup153 levels (Fig. 1D; Supplemental Fig. S1D). Together, these results show that the observed induction of early differentiation is caused by Nup153 deficiency. Interestingly, Nup153 expression was down-regulated upon differentiation of mESCs into neural precursor (NeuP) cells (Fig. 1F), further suggesting that Nup153 expression correlates with the undifferentiated state.

Importantly, knockdown of Nup153 did not significantly alter the expression levels of the core pluripotent factors Oct4, Nanog, and Klf4 as judged by qRT-PCR and Western blot analysis (Fig. 1A,G). These results suggest that the induction of differentiation observed in Nup153-depleted mESCs is not caused by the down-regulation of markers of the undifferentiated state, which prompted us to test whether Nup153 might be involved in the regulation of differentiation genes.

\section{Nup153 depletion induces expression of developmental genes}

To gain insight into the molecular mechanisms by which Nup153 maintains the mESC pluripotent state, we ana- lyzed global gene expression profiles using next-generation sequencing of total RNA (RNA-seq). Compared with mock-depleted cells, knockdown of Nup153 resulted in up-regulation and down-regulation (fold change $\geq 1.5$ and false discovery rate [FDR] $<0.05$ ) of 1234 and 660 genes, respectively (Fig. 2A; Supplemental Table S1). Consistent with the observed morphological changes, early ectodermal differentiation markers such as Pax6, Neurod1, Blbp (also known as Fabp7), Sox11, Nefh, Nrp1, and Reln were up-regulated in Nup153-depleted mESCs (Fig. 2A). A gene ontology (GO) analysis of the list of upregulated transcripts showed a significant enrichment for genes involved in transcription, cell morphogenesis, and neuron development (Fig. 2B).

To determine whether Nup153 deficiency enhanced primarily neuroectoderm differentiation, we differentiated mESCs into NeuP cells and analyzed their gene expression profiles by RNA-seq (Supplemental Fig. S2A; Supplemental Table S2). A significant number of genes that were up-regulated after Nup153 knockdown ( 29\%) were also induced during neural differentiation (Fisher's exact test, $\left.P<10^{-20}\right)$, supporting a role for Nup153 in maintaining a specific subset of early lineage-specific genes in a repressed state (Fig. 2C; Supplemental Fig. S2B). The RNAseq further confirmed that Nup153 knockdown did not affect the levels of the core transcriptional network that maintains the pluripotent ESC phenotype, as judged by
A

-1234 upregulated genes $(>1.5$ fold, $5 \%$ FDR)

.660 downregulated genes (<-1.5 fold, $5 \%$ FDR)

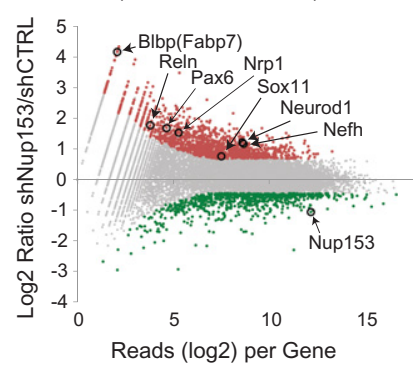

C

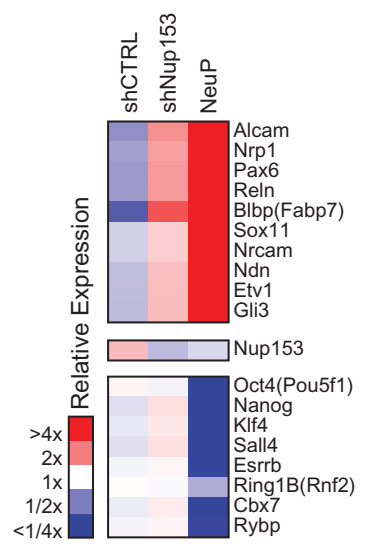

B

GO (Upregulated genes after Nup153 depletion)

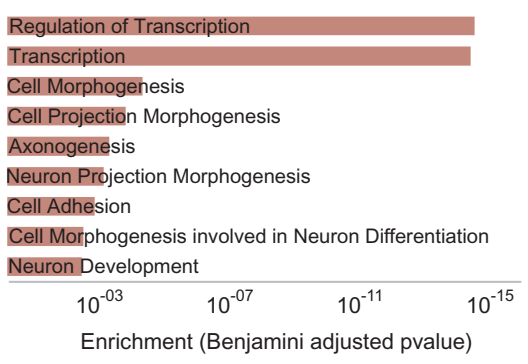

D



E

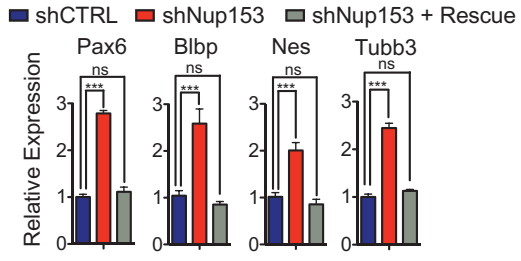

Figure 2. Nup153 depletion causes the induction of developmental gene expression. (A) RNA-seq scatter plot showing the up-regulated and down-regulated genes after $6 \mathrm{~d}$ of Nup153 knockdown. Two biological replicates using independent lentiviral shRNA vectors (shNup153 \#2 and shNup153 \#4) were analyzed, merged, and compared with the control. Arrows indicate representative ectodermal markers up-regulated by Nup153 knockdown. (B) GO analysis of the up-regulated genes upon Nup153 depletion. (C) Heat map from RNA-seq data showing the relative expression of ectodermal markers, pluripotency factors, and PRC1 components in control mESCs, Nup153 knockdown mESCs, and NeuP cells. Nup153 relative expression is shown as control. $(D)$ qRT-PCR analysis of four different ectodermal markers (Pax6, Blbp, Nes, and Tubb3) after $6 \mathrm{~d}$ of Nup153 knockdown. The relative expression levels were normalized to actin and are expressed as fold change relative to the control (shCTRL). The mean $\pm \mathrm{SD}$ from three independent experiments is shown. $P$-values were obtained from Student's $t$-test; $\left({ }^{* * *}\right) P<0.001$. $(E)$ Relative mRNA levels of Pax6, Blbp, Nes, and Tubb3 in control, knockdown, and rescued mESCs measured by qRT-PCR. 
the expression of Oct4, Nanog, Klf4, Sall4, and Esrrb (Fig. 2C). Additional confirmation for the up-regulation of neural-specific genes Pax6, Blbp, Nes, and Tubb3 was obtained by qRT-qPCR analysis (Fig. 2D) and Western blot analysis (Supplemental Fig. S2C).

Importantly, the derepression of developmental genes was rescued by an shRNA-resistant Flag-mCherryNup153 (Fig. 2E), confirming that Nup153's role in repressing early lineage-specific genes is specific. Altogether, we conclude that the early induction of differentiation observed in mESCs upon Nup153 depletion is caused by the up-regulation of early ectodermal lineage-specific genes, implying that Nup153 has an unanticipated role in gene silencing.

\section{Nup153 is essential for mESC pluripotency}

The early induction of differentiation genes observed in Nup153-depleted mESCs suggested that their pluripotent state was compromised, and we therefore tested their capacity to form embryoid bodies (EBs) in vitro and teratomas in vivo. Analysis of ectodermal markers Nes, Tubb3, and Blbp by qRT-PCR in control and Nup153 knockdown EBs at days 8 and 12 demonstrated that these lineage-spe- cific genes were significantly up-regulated in the absence of Nup153 (Fig. 3A). In contrast, the endoderm-specific genes Gata4, Sox17, and Rbp were markedly down-regulated in Nup153 knockdown EBs (Fig. 3B). The induction of mesoderm-specific genes like Isl-1, Brach-T, and a-Sma (smooth muscle actin) during EB differentiation occurred at comparable levels in both control and Nup153-depleted EBs (Supplemental Fig. S3A). Further expression analysis of ectodermal markers by immunostaining in Nup153depleted EBs showed a marked increase of neural progenitor cells (i.e., stained with Blbp and Nes) and neurons (i.e., stained with Tuj1) relative to control EBs (Fig. 3C). In contrast, the immunostaining for the endodermal genes Gata4 and Foxa2 in Nup153 knockdown EBs showed that these EBs did not form the typical outer layer of primitive endoderm present in control EBs (Fig. 3D; Supplemental Fig. S3C). As expected, the overall number of mesodermal cells was similar between the Nup153-depleted and control EBs (Supplemental Fig. S3B). Furthermore, Nup153depleted mESCs gave rise to teratomas with abundant neural differentiation (stained with GFAP [glial fibrillary acidic protein] and Tuj1) when compared with teratomas originated from control mESCs (Fig. 3E; Supplemental Fig. S3D). Altogether, these results show that down-

A
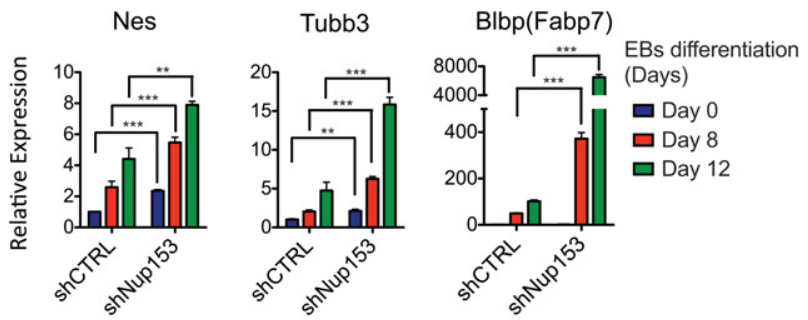

B
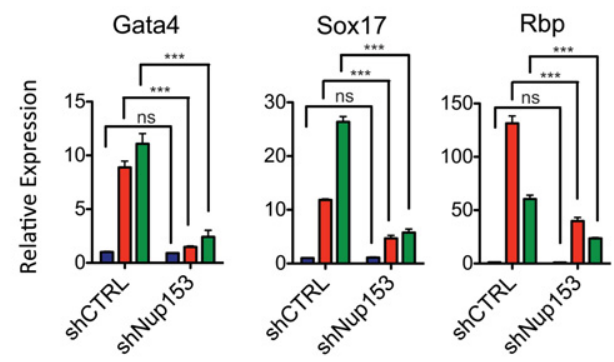

EBs differentiation (Days)

$\square$ Day 0

Day 8 ㅁay 12

C
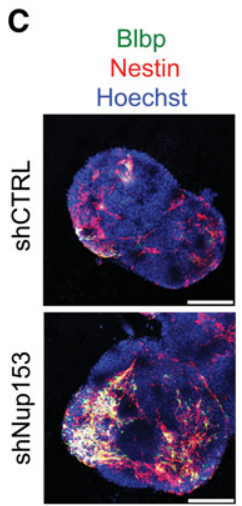

Tuj1(Tubb3) Hoechst

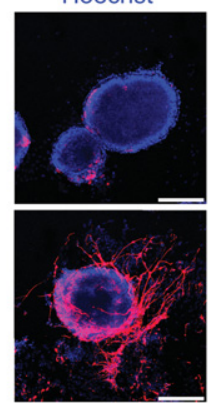

D

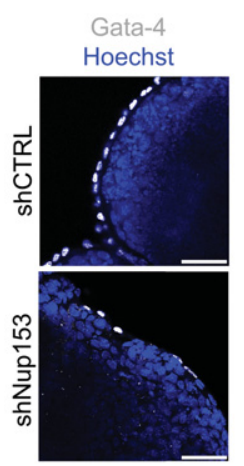

E

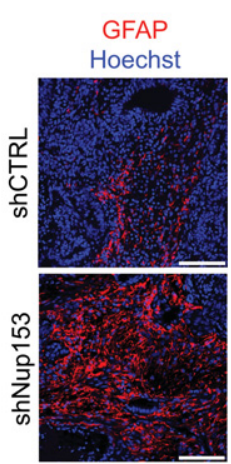

Figure 3. Nup153 is required for mESC pluripotency. (A) qRT-PCR analysis showing the relative expression levels of representative ectodermal markers in control and Nup153 knockdown EBs at days 0, 8, and 12 after LIF removal. The relative expression levels were normalized to actin and are expressed as fold change relative to the respective control. The mean \pm SD from three independent experiments is shown. $P$ values were obtained from Student's $t$-test; $\left({ }^{* *}\right) P<$

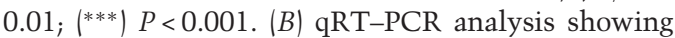
the relative expression levels of representative endodermal markers in control and Nup153-depleted EBs at days 0,8 , and 12 of EB differentiation. (C) Immunofluorescence for the ectodermal markers Blbp, Nes, and Tuj1 in control and Nup153-depleted EBs at day 12 of EB differentiation. Bars, $200 \mu \mathrm{m}$. (D) Immunofluorescence for the endodermal marker Gata4 in control and Nup153-depleted EBs at day 12 of EB differentiation. Bars, $50 \mu \mathrm{m}$. (E) Representative immunofluorescence data for the ectodermal marker GFAP on paraffin-embedded sections of teratomas originated from control and Nup153-depleted mESCs. Bars, 100 $\mu \mathrm{m}$. 
regulation of Nup153 induces neuroectoderm differentiation that derives from a propensity already detected in the undifferentiated state.

\section{The premature induction of differentiation genes is not caused by global defects in nuclear transport}

Originally identified as a component of the NPC, Nup153 has been shown to be involved in RNA export (Bastos et al. 1996; Ullman et al. 1999; Dimaano et al. 2001) and protein import (Shah et al. 1998; Walther et al. 2001; Sabri et al. 2007). However, recent findings in Drosophila suggest a role in transcription regulation that is independent of its role as a constituent of NPCs (Vaquerizas et al. 2010). To determine whether the observed early differentiation phenotype in Nup153-depleted mESCs was a result of de- fects in nuclear trafficking, we first tested the nuclear localization of the key pluripotency factors Oct4 and Sox 2. In both cases, nuclear stainings for both Oct4 and Sox 2 were indistinguishable between control and Nup153depleted mESCs (Fig. 4A; Supplemental Fig. S4A). Furthermore, immunofluorescence fluorescent RNA in situ hybridization (IF-RNA-FISH) analysis using an oligo dT probe in Nup153 knockdown mESCs did not show a significant nuclear accumulation of poly $(A)^{+}$RNAs (Fig. 4B).

Nup153 has been shown to interact with Nup50, and both NPC components as a complex have been implicated in nuclear transport (Matsuura and Stewart 2005; Makise et al. 2012). To directly test a potential involvement of Nup50 in the observed phenotype, we depleted this Nup in mESCs (Fig. 4C; Supplemental Fig. S4B) but did not detect induction of early differentiation (Fig. 4F;
A

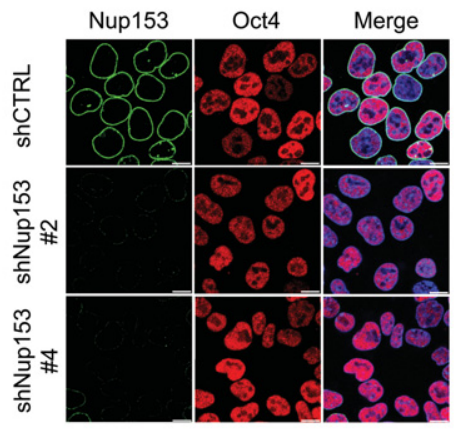

C
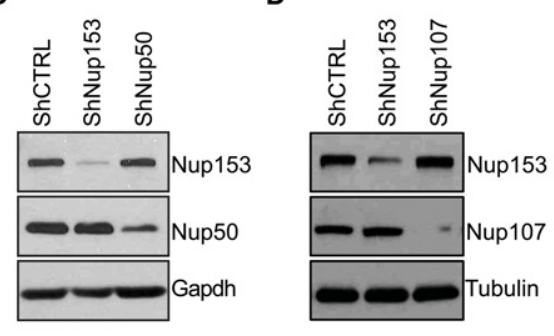

$\mathbf{F}$

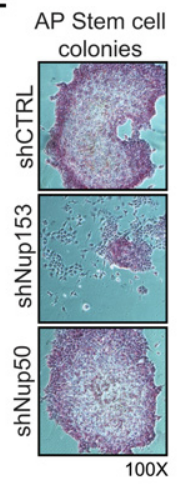

G

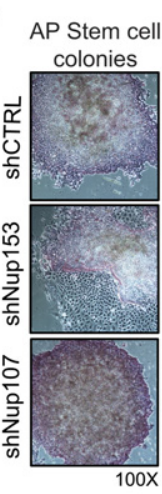

B

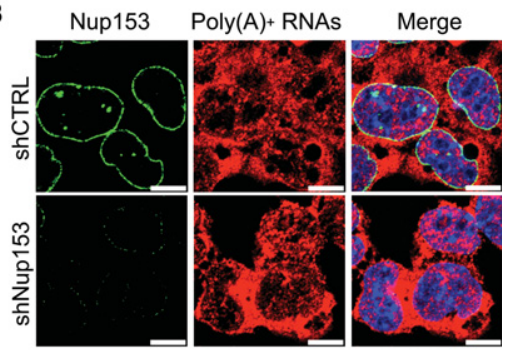

E

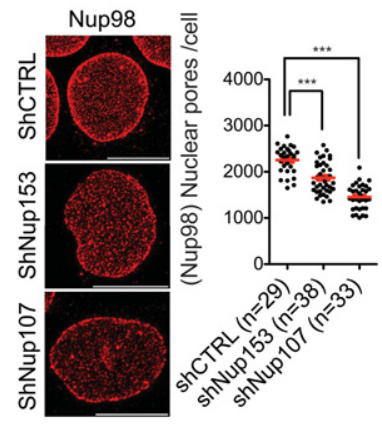

H

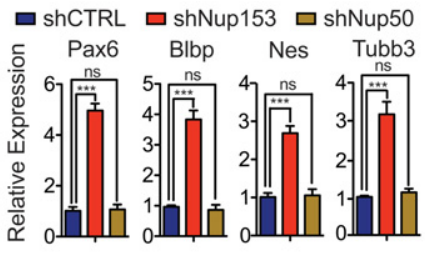

I

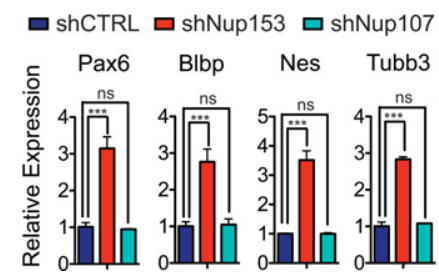

Figure 4. Nup153-mediated silencing of specific developmental genes in $\mathrm{mESCs}$ seems to be independent of its roles in global nuclear transport. (A) Immunofluorescence for the pluripotency marker Oct4 in control and Nup153 knockdown mESCs after $6 \mathrm{~d}$ of Nup153 knockdown. Bars, $10 \mu \mathrm{m}$. (B) Representative image from a Nup153 IF-RNA-FISH for poly(A) ${ }^{+}$ RNAs in control and Nup153 knockdown mESCs after $6 \mathrm{~d}$ of Nup153 depletion. Bars, $10 \mu \mathrm{m} .(C)$ Western blot analysis for Nup153 and Nup50 in whole-cell extracts from control, Nup153 knockdown, and Nup50 knockdown mESCs upon $5 \mathrm{~d}$ of depletion. Gapdh was used as the loading control. (D) Western blot analysis for Nup153 and Nup107 in whole-cell extracts from control, Nup153 knockdown, and Nup107 knockdown mESCs upon $7 \mathrm{~d}$ of depletion. Tubulin was used as the loading control. $(E)$ NPCs were stained and visualized by superresolution microscopy, and individual pores were counted for each nucleus. $N \approx 30$. Maximum projection of representative reconstructed images (left panel) and total number of single pores per cell (right graph) from control, Nup153 knockdown, and Nup107 knockdown nuclei. $P$-values were obtained from Student's $t$-test; $\left.{ }^{* * *}\right) P<0.001$. $(F)$ Representative phase-contrast microscopy images of control, Nup153 knockdown, and Nup50 knockdown AP stem cell colonies. Original magnification, 100×. (G) Representative phase-contrast microscopy images of control, Nup153 knockdown, and Nup107 knockdown AP stem cell colonies. Original magnification, 100×. (H) qRT-PCR analysis of four different ectodermal markers in control, Nup153 knockdown, and Nup50 knockdown mESCs after $5 \mathrm{~d}$ of knockdown. The relative expression levels were normalized to actin and are expressed as fold change relative to the control (shCTRL). (I) qRT-PCR analysis of four different ectodermal markers in con-

trol, Nup153 knockdown, and Nup107 knockdown mESCs after $7 \mathrm{~d}$ of knockdown. The relative expression levels were normalized to actin and are expressed as fold change relative to the control (shCTRL). The mean \pm SD from three independent experiments is shown. $P$ values were obtained from Student's $t$-test; $(* * *) P<0.001$. 
Supplemental Fig. S4E) or up-regulation of lineage-specific genes (Fig. 4H). These findings suggest that the observed differentiation phenotype is unlikely to be the result of global nuclear transport defects.

However, we did observe a modest reduction of NPC number $(\sim 17 \%)$ in Nup153-depleted cells (Fig. 4E). Since NPCs have recently been shown to be involved in chromatin organization and genome function (Pascual-Garcia and Capelson 2014), we wanted to directly test whether NPC numbers are critical for mESC pluripotency. To do this, we depleted Nup107 (a major component of the large subcomplex Nup107/160, whose presence is required for NPC assembly) (Fig. 4D; Supplemental Fig. S4C; Walther et al. 2003), which resulted in a $37 \%$ decrease in the total number of NPCs per nucleus (Fig. 4E; Supplemental Fig. S4D). Similar to the depletion of Nup50, we did not observe an induction of early differentiation in Nup107 knockdown mESCs (Fig. 4G) or a significant up-regulation of ectodermal lineage-specific genes upon deletion of Nup107 (Fig. 4I). Collectively, these data indicate that the Nup153-mediated repression of a subset of developmental genes in mESCs occurs independently of nuclear transport.

\section{Genome-wide mapping of Nup153-binding sites in $\mathrm{mESCs}$ by DamID-seq (DNA adenine methyltransferase identification [DamID] followed by next-generation sequencing)}

We next wanted to explore the possibility that Nup153, a mobile Nup with an intranuclear presence, functions in the regulation of transcriptional programs via physically binding to genomic loci (Vaquerizas et al. 2010). To determine genome-wide binding of Nup153 in mESCs, we used DamID-seq. The DamID technology is based on the ability of the Dam methylase to modify genomic regions that are in molecular contact with Dam fused to a protein of interest in vivo with adenine-6 methylation $\left({ }^{\mathrm{m} 6} \mathrm{~A}\right)$, which is a modification not found in higher eukaryotes (van Steensel and Henikoff 2000; Guelen et al. 2008). We first established a stable mESC line expressing low levels of a chimeric protein consisting of Dam fused to Nup153 (Dam-Nup153) under the heat-shock promoter (in the absence of heat shock). Immunostaining confirmed that this fusion protein was correctly targeted to the NE (Supplemental Fig. S5A). We first validated the DamID-seq method by generating two independent DamID profiles for the core pluripotent factor Oct 4 in mESCs and comparing them with the Oct4 ChIP-seq (chromatin immunoprecipitation [ChIP] followed by next-generation sequencing) profile (Supplemental Fig. S5B). Both stable mESC lines expressing the Dam-Oct4 fusion protein generated reproducible peaks when compared with the GFP-DamID control (Supplemental Fig. S5B). Oct4 DamID-seq peaks highly overlap with Oct4 ChIP-seq peaks, confirming the feasibility of our strategy (Supplemental Fig. S5B,C). Moreover, a motif analysis of Oct4 DamID-seq peaks using a de novo approach has identified the Oct4-binding motif as the most enriched motif $\left(P=1 \times 10^{-71}\right)$, further validating the overall reliability of the DamID-seq approach (Supplemental Fig. S5D).
Using this method, we identified a total of 3517 Nup153 DamID peaks with high confidence (greater than twofold enrichment over Dam-GFP control and FDR $<0.01$ ). An example of a gene locus bound by Nup153 in two independent biological replicates is shown in Supplemental Figure S5E. Strikingly, Nup153 was enriched at the gene body (48\%; gene body being defined as +100 base pairs [bp] from the TSS to $+1 \mathrm{~kb}$ from the termination end site [TES]) (Fig. 5A) and highly enriched around the TSSs of these genes, consistent with a potential role of Nup153 in transcription regulation (Fig. 5B). In addition, $\sim 37 \%$ of Nup153 peaks were present at intergenic regions (Fig. 5A), suggesting that Nup153 might also occupy enhancer regions. GO term enrichment analysis of the 2133 genes bound by Nup153 within $+2 \mathrm{~kb}$ upstream of the TSS to $+1 \mathrm{~kb}$ downstream from the TES showed a significant enrichment for genes involved in cell adhesion, regulation of transcription, embryonic morphogenesis, and chordate embryonic development (Supplemental Fig. 5G). Given that Nup153 depletion in mESCs led to the up-regulation of 1234 genes, we tested whether any of them were direct targets of Nup153. Strikingly, we found that 201 genes had a specific Nup153-binding site within $-2 \mathrm{~kb}$ from the TSS to $+1 \mathrm{~kb}$ from the TES (Fisher's exact test, $P<9.8 \times 10^{-18}$ ) (Fig. 5C). Moreover, it is almost twofold more likely to find a gene bound by Nup153 if it is up-regulated after Nup153 depletion than if it is not regulated (17\% vs. $9 \%)$ (Supplemental Fig. S5F), suggesting that Nup153-directed repression is at least partially mediated by a cis-regulatory mechanism. Interestingly, out of the 201 genes bound by Nup153 and up-regulated after Nup153 depletion, 73 were also up-regulated in NeuP cells $(36 \%)$, suggesting a direct role for Nup153 in the transcriptional repression of a subset of early lineage-specific genes in mESCs. Representative examples of Nup153 peaks at four differentiation genes are shown in Figure 5D. Together, these data suggest that Nup153 mediates the transcriptional silencing of a subset of developmental genes by directly binding to their TSSs in mESCs.

As a mobile NPC component, Nup153 can act both at the NPC and inside the nucleus at sites that are not attached to the NE. Therefore, we wondered whether Nup153-bound genes are preferentially localized at the nuclear periphery. We examined the localization of 10 Nup153 targets whose expression was affected in Nup153 knockdown cells by IF-FISH experiments. We used lamin B2 (Lmnb2) staining as a marker for the NE, quantified the distance of the FISH signals to the NE, and only scored a distance of $0-0.5 \mu \mathrm{m}$ as "peripheral" localization (Fig. 5E,F). Consistent with Nup153 being a mobile Nup, we found six loci to be specifically localized at NPCs and four with clear intranuclear localization (Fig. $5 F)$. Interestingly, genes that were associated with the NE changed to a more intranuclear position in the cells depleted of Nup153 (Fig. 5E,F). This suggests that Nup153 plays a role in proper positioning of a subset of developmental genes at the NPC. However, since nonperipheral targets were also affected under Nup153 knockdown conditions, it appears that gene position relative to the NE is not a critical feature of Nup153-mediated gene silencing. 
A

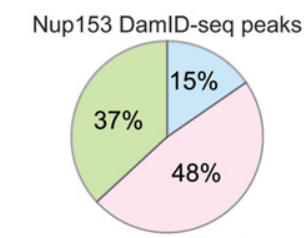

$\square$ Promoter (-2Kb from TSS to $+100 \mathrm{bp}$ from TSS)

$\square$ Gene Body ( $+100 \mathrm{bp}$ from TSS to $+1 \mathrm{~Kb}$ from TES)

Intergenic (<-2Kb from TSS and $>+1 \mathrm{~Kb}$ from TES)
B

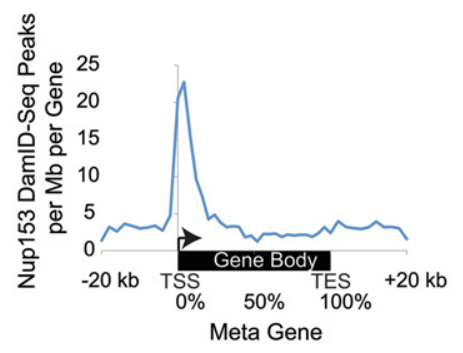

C

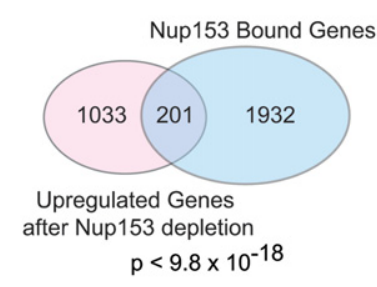

E

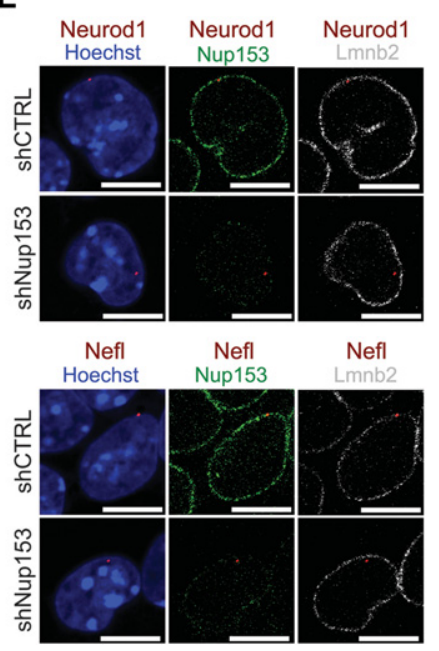

D
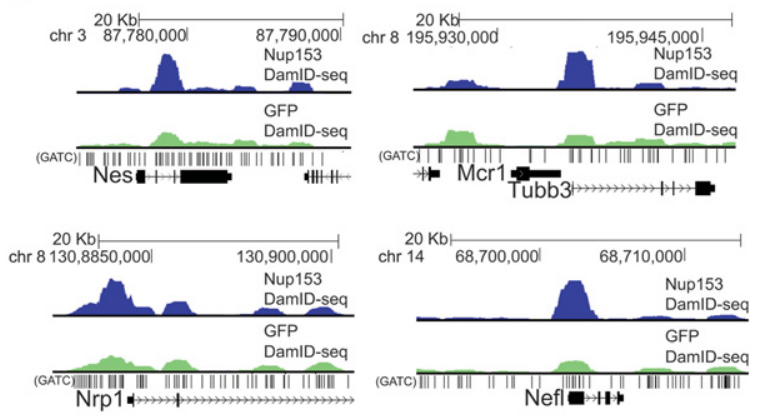

$F$

Distance from the nuclear envelope $(\mu \mathrm{m})$ : $\square 0-0.5$ (nuclear periphery) $\square 0.5-2$

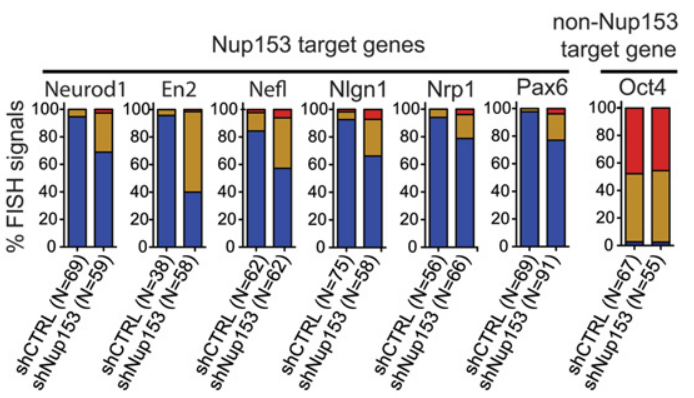

Figure 5. DamID-seq analysis of Nup153-binding sites in mESCs. (A) Distribution of Nup153 DamID-seq peaks relative to RefSeq genes. (B) Global Nup153 gene occupancy profile. Nup153 DamID-seq peaks per megabase per gene for all annotated genes are shown along the transcription units from $20 \mathrm{~kb}$ upstream of the TSS to $20 \mathrm{~kb}$ downstream from the TES. (C) Venn diagram showing the overlap between the induced genes in Nup153 knockdown mESCs and the genes containing a Nup153-binding site within $-2 \mathrm{~kb}$ from the TSS to $+1 \mathrm{~kb}$ from the TES. $P$-value for the overlap was computed using the Fisher's exact test. $(D)$ Examples of Nup153 DamID-seq peaks around the TSSs of four different differentiation genes (Nes, Tubb3, Nrp1, and Nefl) in mESCs. Two independent biological replicates for both Dam-Nup153 and Dam-GFP were sequenced and analyzed. For visualization, the biological replicates were merged. A schematic illustration of the genomic structure of the gene loci, its genomic location, and the associated GATC content is also shown. (E) Representative three-dimensional IFFISH images showing the localization of two "peripheral" Nup153 target genes (Neurod1 and Nefl) in control and Nup153-depleted mESCs after $6 \mathrm{~d}$ of knockdown. FISH signals are shown in red, Nup153 staining is shown in green, Lmnb2 staining is shown in gray, and Hoechst is shown in blue. Bars, $10 \mu \mathrm{m} .(F)$ Percentage of FISH signals from several Nup153 target genes localized at 0-0.5 $\mu \mathrm{m}$ (blue), 0.5-2 $\mu \mathrm{m}$ (yellow), or 2-6 $\mu \mathrm{m}$ (red) from the NE (stained with Lmnb2) in control and Nup153-depleted mESCs after $6 \mathrm{~d}$ of knockdown. The Oct4 nuclear localization is shown as an example of a "nonperipheral" localization.

\section{Nup153 and PRC1 interact and co-occupy specific genomic loci}

Next, we wanted to determine the underlying mechanism of Nup153-mediated gene silencing in naïve mESCs. Based on the signature of Nup153 target genes, we focused on the PRC1, which is bound at and represses the activity of key developmental genes in mESCs and at different stages of differentiation (Boyer et al. 2006; Bracken et al. 2006; Ku et al. 2008). Furthermore, the loss of PRC1 results in derepression of lineage-specific genes, induces early differentiation of mESCs, and thus partially phenocopies Nup153 depletion (Leeb and Wutz 2007; van der Stoop et al. 2008; Endoh et al. 2008; Leeb et al. 2010). 
To test this, ChIP-seq was performed for Ring $1 \mathrm{~b}$, the core subunit of all PRC1 complexes, and chromobox 7 (Cbx7), which is the predominant Cbx protein in mESCs that mediates Cbx-containing PRC1 complexes to bind H3K27me3 (Morey et al. 2012). Strikingly, 40\% of the Nup153 peaks overlap with the PRC1 (presence of the catalytic subunit Ring1b) (Fig. 6A). Notably, the vast majority of the overlap occurs exclusively between Nup153 and Ring1b (30\%) (Fig. 6A). The significant overlap between
Nup153 and Ring1b peaks suggests that Nup153 binds several PRC1 genomic loci independently of H3K27me3, since $\mathrm{Cbx} 7$ is not always required for the Ring $1 \mathrm{~b}$ recruitment. Moreover, among the 2133 Nup153-bound genes, 1358 genes (64\%) are co-occupied by Ring1b and/or Cbx7, strongly suggesting that Nup153 and the PRC1 have a large number of common target genes in mESCs (Fig. 6B). Importantly, the overlap between Nup153 and Ring1b occurs primarily at the TSSs of several genes
A

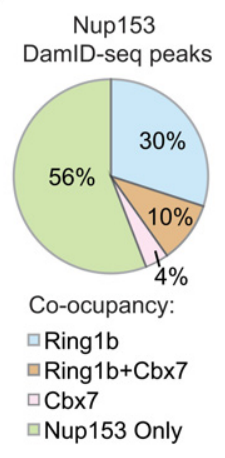

D

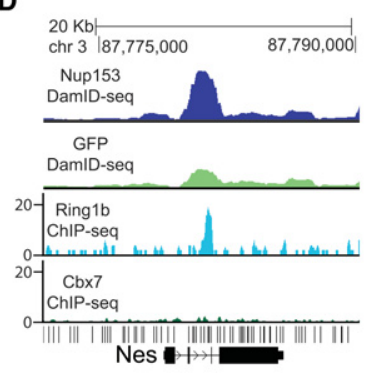

$\mathbf{F}$

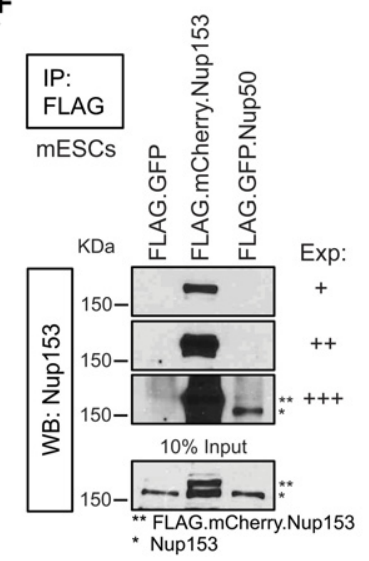

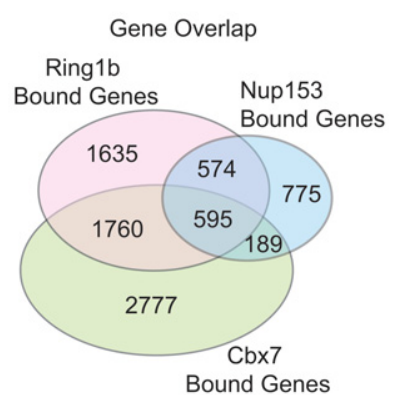

E
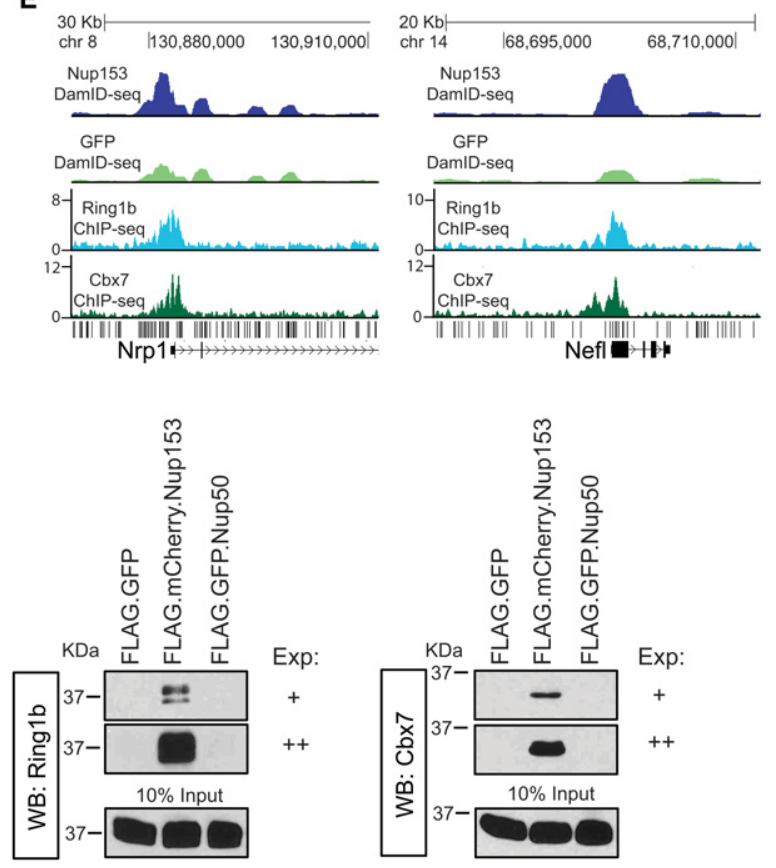

Figure 6. Nup153 and PRC1 components associate with and co-occupy the TSSs of several differentiation genes. $(A)$ Pie chart showing the percentage of Nup153 DamID-seq peaks co-occupied by Ring1b and/or Cbx7 peaks. (B) Venn diagram showing overlap of Nup153-, Ring1b-, and Cbx7-bound genes in mESCs. Bound genes are defined as having occupancy peaks between $-2 \mathrm{~kb}$ from the TSS and $+1 \mathrm{~kb}$ from the TES. (C) Ring1b ChIP-seq read density per TSS at Nup153-bound TSSs (blue) versus unbound TSSs (red). (D) Representative differentiation gene (Nestin) containing a Nup153 DamID-seq peak and a Ring1b ChIP-seq peak at the TSS. For visualization, the biological replicates used to generate the Nup153 and GFP DamID profiles were merged. A schematic illustration of the genomic structure of the gene loci, its genomic location, and the associated GATC content is also shown. (E) Ring1b ChIP-seq, Cbx7 ChIP-seq, and Nup153 DamID-seq profiles at the TSSs of representative developmental genes. $(F)$ Anti-Flag immunoprecipitation of Flag.GFP, Flag.mCherry.Nup153, and Flag.GFP.Nup50 ectopically expressed in mESCs followed by Western blot using antibodies against Nup153, Ring1b, and Cbx7. 
(Fig. 6C). Consistently, Ring $1 \mathrm{~b}$ is significantly enriched at the Nup153-bound TSSs compared with unbound TSSs (Mann-Whitney test, $P<1 \times 10^{-100}$ ) (Supplemental Fig. S6A). Furthermore, the genomic distribution of the Ring $1 \mathrm{~b}$ peaks shows a pattern similar to the one obtained for the Nup153 DamID profile, with $47 \%$ of Ring1b peaks being enriched at the gene body (Supplemental Fig. S6B). GO term enrichment analysis of the genes that contain a Ring $1 \mathrm{~b}$ peak within $-2 \mathrm{~kb}$ from the TSS to $+1 \mathrm{~kb}$ from the TES showed a significant enrichment for genes involved in regulation of transcription, embryonic morphogenesis, chordate embryonic development, and regulation of cell proliferation, categories that were also enriched for the genes bound by Nup153 (Supplemental Fig. S6C). Examples of representative developmental genes co-occupied by Nup153 and Ring1b or Nup153, Ring1b, and Cbx7 are shown in Figure 6, D and E, respectively.

To test whether Nup153 physically associates with PRC1 complexes, we expressed a Flag- and mCherrytagged version of Nup153 in mESCs. The Flag-mCherryNup153 protein was functional, as it properly localized to the NPC and was able to immunoprecipitate Nup50, a protein that requires Nup153 for proper nuclear pore localization (Fig. 6F; Supplemental Fig. S6D; Makise et al. 2012). Strikingly, we found that Flag-mCherry-Nup153 interacted with the PRC1 components Ring $1 \mathrm{~b}$ and $\mathrm{Cbx} 7$ (Fig. 6F). In contrast, Flag-GFP-Nup50 (despite its ability to pull down Nup153) did not interact with Ring1b or Cbx7, suggesting that the interaction between Nup153 and the PRC1 is specific (Fig. 6F). Next, we wanted to test whether the interaction between PRC1 and Nup153 can be detected with endogenous proteins, which is typically more difficult to achieve. We performed coimmunoprecipitation assays using nuclear extracts prepared from control and Nup153-depleted mESCs using antibodies specific for Nup153, Cbx7, Ring1b, and Nup50 (Supplemental Fig. S6E,F). We found that Nup153, but not Nup50, coimmunoprecipitated with $\mathrm{Cbx} 7$ and Ring1b (Supplemental Fig. S6E,F), and Cbx7 was able to pull down Nup153 but not Nup50 (Supplemental Fig. S6E). The levels of pulled-down proteins was low, suggesting that only a subset of the PRC1 complex interacts with Nup153. Altogether, these data suggest that Nup153 and the PRC1 can interact directly or indirectly and occupy common genomic loci in order to maintain the pluripotent state of mESCs.

\section{Nup153 is required for the recruitment of PRC1 complexes to specific differentiation genes}

The factors involved in the recruitment of PRC1 complexes to the TSSs of developmental genes are still poorly defined (Simon and Kingston 2013). Because PRC1 complexes do not contain inherent DNA-specific binding activity, additional proteins might mediate their chromatin recruitment. We therefore wanted to test whether the presence of Nup153 is required for the recruitment of PRC1 to target genes in mESCs. To do this, we generated ChIP-seq profiles for Ring1b in control and Nup153-depleted mESCs. Strikingly, genome-wide Ringlb occupan- cy at Nup153-bound sites was significantly reduced in two independent Nup153-depleted mESCs (Fig. 7A). We also performed ChIP-seq for Ring1b in NeuP cells and found that Ring1b occupancy at Nup153 target sites is strongly decreased (Fig. 7A). Importantly, Ringlb protein levels did not change upon differentiation of mESCs into NeuP cells (Supplemental Fig. S7E). Strikingly, Ringlb occupancy was not significantly reduced after Nup153 depletion when all TSSs were analyzed (Supplemental Fig. S7A). Furthermore, we did not observe a significant decrease of Oct4 occupancy at Nup153-binding peaks in Nup153-depleted mESCs (Supplemental Fig. S7B), suggesting that Nup153 depletion seems to specifically impair the recruitment of Ring1b to Nup153-bound genes. The reduction in Ring1B recruitment in Nup153-depleted mESCs at two representative developmental genes is illustrated in Figure 7B. Importantly, the loss of recruitment of PRC1 to the TSSs of developmental genes in Nup153 knockdown mESCs resulted in their up-regulation, suggesting that Nup153 binding is functionally relevant (Fig. 7B). Among the 201 Nup153-bound genes that increase expression after Nup153 depletion (Fig. 5D), 153 (76\%) were also bound by Ring $1 \mathrm{~b}$ and/or Cbx7. In contrast, a non-Nup153 target, Gata6, did not show a significant change in Ring1b binding after Nup153 knockdown (Supplemental Fig. S7C). Consistent with a transportindependent role of Nup153, we did not observe a significant change in the mRNA levels (Fig. 2D) or protein levels (Fig. 7C) or a change in the subcellular localization of the PRC1 components Ring1b, Cbx7, and RybP in Nup153depleted mESCs compared with control (Fig. 7D; Supplemental Fig. S7D). Altogether, these data indicate that Nup153 plays a previously uncharacterized role in mediating the recruitment of PRC1 complexes to the TSSs of a subset of differentiation-associated genes to silence their expression in mESCs.

\section{Discussion}

In addition to their well-established role in mediating transport, Nups have emerged as potential regulators of developmental gene regulation via direct binding to several developmental loci and association with gene regulatory machinery (Pascual-Garcia and Capelson 2014). However, the molecular mechanism by which Nups mediate transcription and how they control developmental processes, especially in mammals, remained largely unexplored. Here, we report a DamID-seq analysis for one of the components of the NPC, the peripheral Nup Nup153, in mESCs. We found that Nup153 plays a role in the silencing of developmentally regulated genes in mESCs.

In addition to the core gene regulatory circuitry composed of Oct4, Sox2, Nanog, and other transcription factors, polycomb group (PcG) proteins play important roles in repressing early lineage-specific genes in mESCs (Boyer et al. 2006; Bracken et al. 2006; Ku et al. 2008) PcG proteins operate in multicomponent complexes, the best-characterized of which are termed PRC1 and PRC2. These complexes catalyze defined histone tail modifications, with PRC1-type complexes monoubiquitylating histone H2A 
A

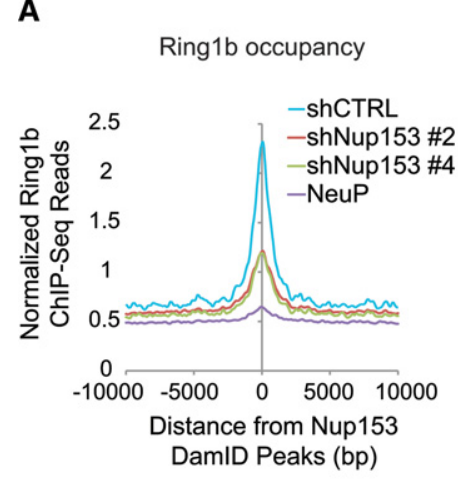

C

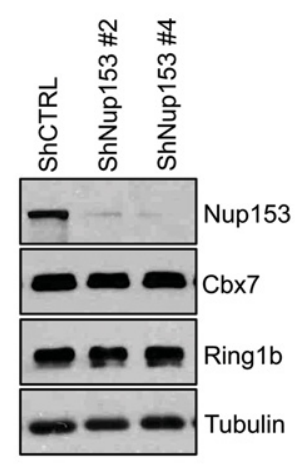

B

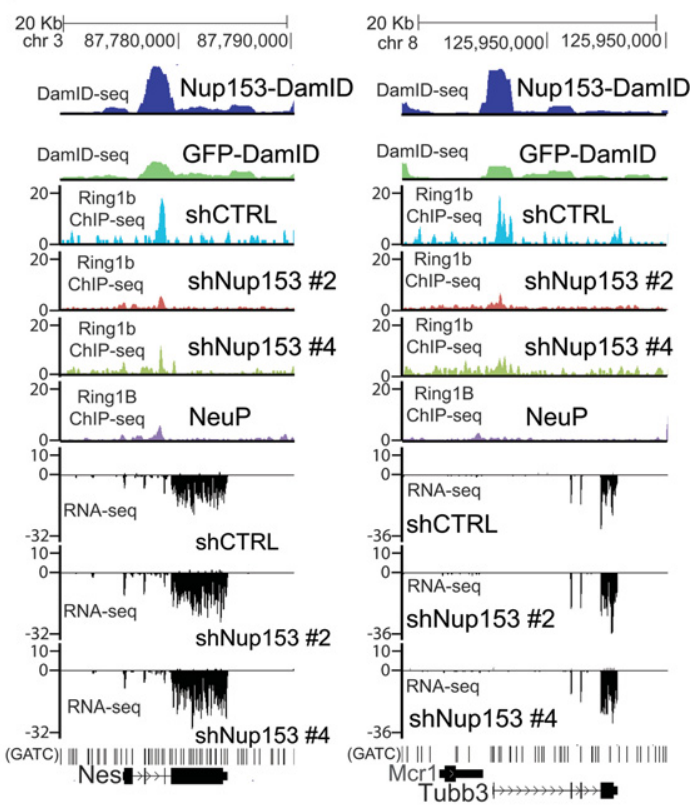

D

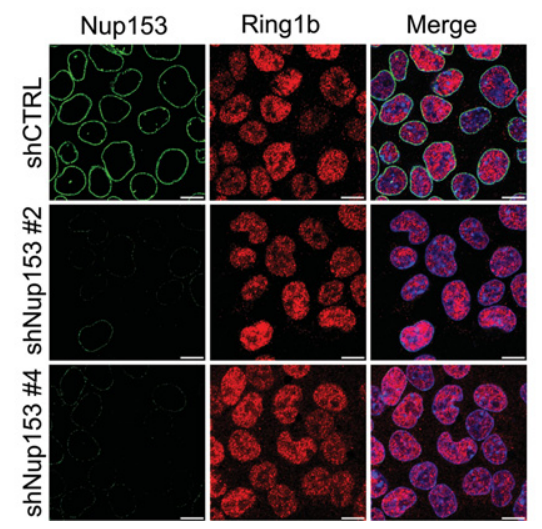

Figure 7. Nup153 Depletion impairs the recruitment of PRC1 to chromatin. (A) Genome-wide occupancy of Ring1b at all identified Nup153 DamID-seq peaks in control mESCs, Nup153 knockdown mESCs, and NeuP cells. (B) Ring1b ChIP-seq, Nup153 DamID-seq, and RNA-seq profiles at two representative differentiation genes in control mESCs, Nup153 knockdown mESCs, and NeuP cells are shown. For visualization, the biological replicates used to generate the Nup153 and GFP DamID profiles were merged. The genomic location of the gene loci and the associated GATC content are also shown. The $Y$-axis in all Ring $1 \mathrm{~b}$ ChIP-seq and RNA-seq profiles shown in both gene loci represents the normalized number of reads. (C) Western blot analysis for Nup153, Cbx7, and Ring1b in whole-cell extracts from mESCs after $6 \mathrm{~d}$ of Nup153 depletion. Tubulin was used as the loading control. $(D)$ Immunofluorescence for Ring $1 \mathrm{~b}$ in control and Nup153 knockdown mESCs after $6 \mathrm{~d}$ of Nup153 depletion. Bars, $10 \mu \mathrm{m}$.

(H2AK119ub1) and PRC2 methylating histone H3 Lys27 (H3K27me3) (Simon and Kingston 2013). Although the function of the PRC1 and PRC2 complexes in repressing developmental genes and maintaining the undifferentiated state of mESCs has been well documented, the mechanisms by which they are recruited to target sites is not well understood. In this study, we provide evidence that Nup153 interacts with the PRC1 and is required for its recruitment to early differentiation genes. Interestingly, at several of these genomic sites, this recruitment seems to be independent of PRC2 and H3K27me3, since Cbx7, the predominant $\mathrm{Cbx}$ protein that helps recruit $\mathrm{Cbx}$-containing PRC1 subtypes to H3K27me3 (Morey et al. 2012), is not present. Supporting our findings, it has been shown that the PRC1 can be recruited to chromatin independently of PRC2 (Schoeftner et al. 2006; Tavares et al. 2012). Moreover, examples of PRC1 targeting through interactions with DNA-binding factors such as Runx1, Rest, and, more recently, Kdm $2 \mathrm{~b}$ have emerged (Dietrich et al. 2012; Farcas et al. 2012; Yu et al. 2012; He et al. 2013; Wu et al. 2013).

Together, our data are consistent with the existence of PRC2-independent mechanisms and suggest that Nup153 might participate in the recruitment of PRC1 complexes to developmental genes. Importantly, not all PRC1 target sites are co-occupied by Nup153, suggesting 
that Nup153 is only required for a subset of genes and that additional recruitment mechanisms are likely to exist to achieve silencing of developmental genes. However, the Cavalli group (Gonzalez et al. 2014), using a microscopybased genome-wide RNAi screen, has recently identified Nup153 as a regulator of the nuclear organization of PcG proteins in the Drosophila nucleus, further highlighting the importance of Nup-mediated PRC1 function.

It is worth mentioning that at early stages of Nup153 knockdown, the pluripotency markers Oct4, Nanog, and Klf4 are not affected, yet cells are clearly no longer pluripotent, as they induce a subset of ectodermal genes. This suggests that in the absence of Nup153, ESCs are pushed into an "intermediate" state that eventually leads to loss of pluripotency and the full induction of differentiation. Since Nup153 is directly involved in regulating the expression of a subset of ectodermal lineage-specific genes in mESCs, we conclude that decreased levels of Nup153 allow the expression of differentiation factors that will eventually override pluripotency factors for establishing cellular identity. It is important to mention that the AP staining experiments are performed at least $25 \mathrm{~d}$ after shRNA infection; at this time point, a vast majority of the Nup153-depleted cells are not at this intermediate state anymore and, as expected, do not express pluripotency factors (data not shown). In fact, the morphology of a significant number of Nup153-depleted cells in our AP staining experiments is indicative of cells that are fully differentiated.

Also, in Drosophila, Nup153 has been shown to bind to large chromatin domains (extending $10-500 \mathrm{~kb}$ ) to define transcriptionally active regions in the genome /Vaquerizas et al. 2010). This is in clear contrast to our findings in ESCs where Nup153 occupies the TSSs of a subset of developmental genes. This suggests that while the role of Nup153 in gene regulation is evolutionarily conserved, it might have developed species-specific functions. Alternatively, this discrepancy between the Drosophila and mESC data may be a reflection of Nup153's ability to function in gene activation and gene repression during different developmental stages. Thus, it will be interesting to determine whether Nup153 has similar function in polycomb-mediated gene silencing in flies or can play a role in gene activation at later stages of mammalian development. Supporting Nup153's role as a nuclear platform for gene activation in Drosophila, it has been reported that Nup153 interacts with the dosage compensation complex and is necessary for the twofold hyperactivation of the male X chromosome (Mendjan et al. 2006). Based on our results, it is attractive to speculate that Nup153 could play a not yet identified role in recruiting epigenetic silencers, including PRC1 complexes, during X-chromosome inactivation in mammalian differentiated female cells. Supporting our findings that associate a component of the NPC with gene silencing in mESCs, a recent study identified a novel role for the yeast Nup Nup170p in silencing genomic regions that contain ribosomal proteins and subtelomeric genes through its association with the RSC chromatin remodeling complex and the silencing factor Sir4p (Van de Vosse et al. 2013). Furthermore, a ge- nome-wide study in human cells has revealed that several binding sites of another component of the NPC, Nup93, were associated with genomic regions enriched with repressive histone modifications (Brown et al. 2008).

Despite the role of Nup153 in nuclear transport (Bastos et al. 1996; Shah et al. 1998; Ullman et al. 1999; Dimaano et al. 2001; Walther et al. 2001; Sabri et al. 2007), our findings provide another example of the transport-independent role of Nups in developmental gene regulation. We found that the nuclear localization of several nuclear proteins such as Oct4, Sox2, Ring1b, Rybp, and Cbx7 was not significantly affected in Nup153 knockdown mESCs, arguing against a global nuclear import defect. Moreover, we did not observe a significant defect of the nuclear export of poly(A) ${ }^{+}$RNAs upon Nup153 depletion. Similar to our results, it has been reported that human cancer cells with Nup153 deficiency can still carry out efficient global nucleocytoplasmic transport, including nuclear import of the glucocorticoid receptor, nuclear export of a Revchimera protein, and nuclear export of poly $(\mathrm{A})^{+}$RNAs (Mackay et al. 2009). These results suggest that Nup153 (and possibly other Nups, such as Nup98) might primarily function as gene regulators and not as mediators of transport, at least in specific cell types such as ESCs.

In organisms like yeast, Nup-chromatin interactions are likely to be restricted to the NE (Van de Vosse et al. 2011). However, in higher eukaryotes, the ability of several Nups to populate the nucleoplasm allows these proteins to interact with several genomic loci throughout the nucleoplasm (Rabut et al. 2004; Van de Vosse et al. 2011; PascualGarcia and Capelson 2014). Strikingly, it has been shown that several of these nucleoplasmic Nup-chromatin associations occur at developmentally regulated genes, suggesting that specific Nups could also behave as nuclear platforms inside the nucleoplasm for setting developmental transcriptional programs (Capelson et al. 2010; Kalverda et al. 2010; Liang et al. 2013). Accordingly, our data show that Nup153-genome interactions can occur both at the NPC and away from the NE. Since Nup153 depletion affects the transcriptional status of both peripheral and nonperipheral target genes, our data do not support the notion that localization at the NPC is critical for the silencing of Nup153 target genes. However, future work will be necessary to determine whether NPC-associated genes are subject to a distinct set of regulatory modalities when compared with intranuclear genes.

In summary, the data presented in this study not only reveal a critical function for Nup153 in mediating the undifferentiated state of mESCs but also establish an unexpected nuclear transport-independent role of Nup153 in gene silencing and thus introduce new mechanistic directions for elucidating the role of Nups during mammalian development.

\section{Materials and methods}

\section{Cell culture and differentiation}

E14, 16.7, and D3 mESCs were cultured on irradiated mouse embryonic fibroblasts in ES medium: knockout DMEM (Gibco), 
15\% Gibco knockout serum replacement (Gibco), MEM nonessential amino acids (Gibco), and mLIF (ESGRO, EMD Millipore). The medium was changed every day, and cells were split every 3$4 \mathrm{~d}$. StemTAG AP staining kit (Cell Biolabs) was used to identify positive AP mESC colonies. To induce differentiation, mESCs were grown in low-attachment plates (Corning Life Sciences) with ES medium without mLIF to allow for EB formation. Differentiation of EBs to NeuP cells was induced by the addition of $0.5 \mu \mathrm{g} / \mathrm{mL}$ mouse Noggin in N2 medium (DMEM/F12/glutamax [Invitrogen]), 1\% B27 supplement (Invitrogen), and 1\% N2 supplement (Invitrogen). NeuP cells were grown in N2 medium plus $20 \mathrm{ng} / \mathrm{mL}$ FGF2 and $20 \mathrm{ng} / \mathrm{mL}$ EGF.

\section{shRNA lentiviral and Nup153 overexpression vectors}

shNup153 \#2 (TRCN0000102396), shNup153 \#4 (TRCN0000102 398), shNup107 (TRCN0000099711), and shNup50 (TRCN0 000102048) MISSION shRNA plasmid DNAs were obtained from Sigma. The shCTRL (SHC002) nontarget control was also purchased from Sigma. To generate a Nup153 expression vector, full-length Nup153 was PCR-amplified and cloned into the BglII/ KpnI sites of the pmCherry-C1 vector. Mutations were targeted to the 21-bp shNup153 \#2 and shNup153 \#4 sites, changing the third nucleic acid of the codons in that region without altering the amino acid sequence. shRNA-resistant Flag.mCherryNup153 and Flag.GFP were amplified by PCR and cloned into the pLV-XEP expression vector using NotI/MluI sites under the EF1 $\propto$ promoter.

\section{shRNA production, infection, and selection}

Viral particles were produced in $293 \mathrm{~T}$ cells by cotransfection with the plasmids pMDLg/pRRE, pRSV/REV, and pMD2.G and concentrated by ultracentrifugation. Highly concentrated viral stocks $\left(1 \times 10^{10}\right.$ to $\left.5 \times 10^{10} \mathrm{TU} / \mathrm{mL}\right)$ were generated in the Viral Vector Facility of the Salk Institute. Approximately $5 \times 10^{6}$ mESCs per $10-\mathrm{cm}$ plate were infected with lentivirus at a multiplicity of infection (MOI) of 50. At $48 \mathrm{~h}$ after transduction, $2 \mu \mathrm{g} /$ $\mathrm{mL}$ puromycin was added to the ES medium to select transduced mESCs. Stable knockdown mESCs were harvested for RNA, Western blot, immunofluorescence, and ChIP-seq after at least $4 \mathrm{~d}$ of selection.

\section{$R N A$ extraction and $q R T-P C R$}

RNA was extracted from mESCs and NeuP cells using Trizol (Ambion) and column-purified using RNeasy kit (Qiagen). RNA was subsequently reverse-transcribed using the QuantiTect reverse transcription kit (Qiagen), and cDNA was subjected to qPCR using SYBR Green PCR master mix (Applied Biosystems). Primer sequences are available in Supplemental Table S3.

\section{Western blot and immunofluorescence}

For protein extracts, mESCs were resuspended in RIPA lysis and extraction buffer (25 mM Tris- $\mathrm{HCl}$ at $\mathrm{pH} 7.6,150 \mathrm{mM} \mathrm{NaCl}$, $1 \%$ NP-40, $1 \%$ sodium deoxycholate, $0.1 \%$ SDS), incubated for $30 \mathrm{~min}$ on ice, and passed five to 10 times through a 27 -gauge syringe. Protein concentration was determined using the BCA reagent (Pierce) and normalized, and $4 \% \beta$-mercaptoethanol and $0.01 \%$ bromophenol blue were added. For Western blot analysis, $40-80 \mu \mathrm{g}$ of protein was resolved in SDS-PAGE gels and transferred to Immobilon-FL membranes (Millipore). Membranes were blocked with PBS and $0.05 \%$ Tween $(\mathrm{PBS}-\mathrm{T})+5 \%$ nonfat milk for $1 \mathrm{~h}$, washed, and incubated with primary antibody over- night at $4^{\circ} \mathrm{C}$. The secondary antibody was added after three washes with PBS-T for $1 \mathrm{~h}$ at room temperature. Membranes were visualized by enhanced chemiluminescence.

For immunofluorescence, mESCs were cultured on Matrigelcoated coverslips for at least $24 \mathrm{~h}$, fixed in $1 \times$ PBS and $4 \%$ PFA for 10 min, permeabilized with $1 \times$ PBS and $0.5 \%$ Triton X-100 for $5 \mathrm{~min}$ on ice, and blocked in $1 \times$ PBS and $1 \%$ BSA for $15 \mathrm{~min}$. The coverslips were then incubated with primary antibody diluted in $1 \times \mathrm{PBS}$ and $1 \%$ BSA overnight at $4^{\circ} \mathrm{C}$ in a humid chamber. The next morning, the coverslips were washed in $1 \times$ PBS, incubated with secondary antibody for $2 \mathrm{~h}$, washed in $1 \times$ PBS, stained with Hoechst, and mounted in VectaShield (Vector Laboratories). The images were acquired using a Zeiss LSM 710 confocal microscope. Images were analyzed and prepared for presentation in Photoshop.

\section{NPC counting}

NPCs were stained with antibodies against Nup98 and Nup153 overnight at $4^{\circ} \mathrm{C}$ in a humid chamber, washed, and secondarystained for $1 \mathrm{~h}$. Coverslips were mounted with Prolong Gold (Invitrogen) and allowed to cure for $24 \mathrm{~h}$ at room temperature. Nuclei were then imaged with a Zeiss Elyra structured illumination superresolution microscope. After three-dimensional reconstructions, pore numbers were quantitated using the spot finder tool in Imaris (Bitplane), and surface areas were quantitated using the surfaces tool. Around 30 nuclei were quantitated from at least two independent experiments.

\section{Preparation of nuclear extracts for immunoprecipitation}

Flag.GFP-, Flag.mCherry.Nup153-, and Flag.GFP.Nup50-expressing mESCS; control mESCs; or Nup153-depleted mESCs $\left(2 \times 10^{8}\right)$ were harvested, washed with PBS, resuspended in buffer A (10 $\mathrm{mM}$ HEPES at $\mathrm{pH} 7.6,1.5 \mathrm{mM} \mathrm{MgCl} 2,10 \mathrm{mM} \mathrm{KCl}, 0.5 \mathrm{mM}$ $\mathrm{DTT}+$ protease inhibitor cocktail [Roche]), and incubated for 10 min on ice. Nuclei were spun down at $13,000 \mathrm{rpm}$ for $10 \mathrm{~min}$ and resuspended in hypotonic buffer $\mathrm{C}(20 \mathrm{mM}$ HEPES at $\mathrm{pH}$ 7.6, $25 \%$ glycerol, $420 \mathrm{mM} \mathrm{NaCl}, 1.5 \mathrm{mM} \mathrm{MgCl}_{2}, 0.2 \mathrm{mM}$ EDTA, 0.5 mM DTT + protease inhibitor cocktail [Roche]). The nuclear extraction was performed for $30 \mathrm{~min}$ at $4{ }^{\circ} \mathrm{C}$ with light shaking. After centrifugation at 13,000 rpm for $15 \mathrm{~min}$, the nuclear extracts (supernatant) were introduced into dialysis bags and dialyzed in $1 \mathrm{~L}$ of dialysis buffer $\mathrm{D}(20 \mathrm{mM}$ HEPES at $\mathrm{pH} 7.6$, $25 \%$ glycerol, $100 \mathrm{mM} \mathrm{KCl}, 1.5 \mathrm{mM} \mathrm{MgCl}_{2}, 0.2 \mathrm{mM}$ EDTA, 0.5 $\mathrm{mM}$ DTT $+0.2 \mathrm{mM}$ PMSF) for $2 \mathrm{~h}$ at $4^{\circ} \mathrm{C}$. The insoluble fraction was removed by centrifugation at 13,000 rpm for $15 \mathrm{~min}$, and supernatants were used for immunoprecipitation.

\section{Immunoprecipitation}

Flag.GFP, Flag.mCherry.Nup153, and Flag.GFP.Nup50 immunoprecipitations were performed using an anti-Flag M2 affinity gel (Sigma-Aldrich). Flag M2 beads were equilibrated in buffer C100* (20 mM HEPES at pH 7.6, 10\% glycerol, $100 \mathrm{mM} \mathrm{KCl}, 1.5$ $\mathrm{mM} \mathrm{MgCl} 2,0.2 \mathrm{mM}$ EDTA, $0.02 \%$ NP40, $0.5 \mathrm{mM} \mathrm{DTT}+$ protease inhibitor cocktail [Roche]), blocked with 5\%BSA for $1 \mathrm{~h}$, and washed three times with buffer C-100*. Approximately $800 \mu \mathrm{L}$ of each nuclear extract at a concentration of $2 \mathrm{mg} / \mathrm{mL}$ was incubated with $60 \mu \mathrm{L}$ of Flag M2 beads for $4 \mathrm{~h}$ at $4^{\circ} \mathrm{C}$ in nonstick microcentrifuge tubes. Immunoprecipitations were washed with C-100* five times for $5 \mathrm{~min}$ at $4^{\circ} \mathrm{C}$ and eluted from beads by incubation with SDS loading dye for $1 \mathrm{~h}$ at room temperature. Ten percent input and 30\% Flag.GFP, Flag.mCherry.Nup153, and Flag.GFP.Nup50 immunoprecipitations were analyzed by SDSpolyacrylamide gel electrophoresis. 
For the immunoprecipitation of native proteins, nuclear extracts were prepared from $2 \times 10^{8}$ control or Nup153 knockdown mESCs. Ten micrograms of each specific antibody was added to $\sim 800 \mu \mathrm{L}$ of each nuclear extract at a concentration of $2 \mathrm{mg} / \mathrm{mL}$ and incubated overnight at $4^{\circ} \mathrm{C}$. The next morning, we added 60 $\mu \mathrm{L}$ of preblocked Protein A Dynabeads (Life technologies) to the nuclear extracts and incubated it on a rotator for $1 \mathrm{~h}$ at $4^{\circ} \mathrm{C}$. Immunoprecipitations were washed with C-100* five times for 5 min at $4^{\circ} \mathrm{C}$ and eluted from beads by incubation with SDS loading dye for $1 \mathrm{~h}$ at room temperature. Ten percent of input and $30 \%$ of each immunoprecipitation and the appropriate control coimmunoprecipitations were analyzed by SDS-polyacrylamide gel electrophoresis.

\section{IF-RNA-FISH}

mESCs were fixed, immunostained with mouse anti-Nup153 (1:1), fixed, permeablized on $1 \times$ PBS and $0.2 \%$ Trion X-100, washed with $2 \times \mathrm{SSC}$, and incubated for $2 \mathrm{~h}$ at $37^{\circ} \mathrm{C}$ on a probe $\operatorname{mix}(2 \times$ SSC, $10 \%$ formamide, $0.02 \%$ RNase-free BSA, $10 \%$ dextran, $2 \mathrm{mM}$ vanadyl-ribonucleoside complexes, $0.4 \mu \mathrm{g} / \mu \mathrm{L}$ carrier RNA) containing the oligonucleotide polyT50 probe [Alexa 568; $\mathrm{T}(50)$, Invitrogen]. After hybridization, the coverslips were washed three times at $37^{\circ} \mathrm{C}$ with $2 \times$ SSC and $10 \%$ formamide, stained with Hoechst, and mounted in VectaShield (Vector Laboratories).

\section{Stem cell subcutaneous injection for teratoma formation}

mESC suspension $(0.2 \mathrm{~mL})$ containing $5 \times 10^{7}$ cells was injected subcutaneously into the dorsal surface (neck) of an 8- to 10-wkold NSG mouse from The Jackson Laboratory. The procedure was performed under isoflorane anesthesia. A $1-\mathrm{cm}^{2}$ area of the mouse's back was shaved, swabbed with Nolvasan (chlorhexidine) solution, and then wiped with alcohol, and the cell suspension was injected using a 21-gauge needle attached to Hamilton syringe.

\section{Immunohistochemistry (IHC) and IF on teratoma sections}

For IF, $5 \mu \mathrm{m}$ of paraffin-embedded teratoma sections were pretreated with a proteinase $\mathrm{K}$ solution prior to incubation with rabbit anti-GFAP (1:1000; Dako). After washing, primary antibody was detected using donkey anti-rabbit-Cy3 (1:250; Jackson ImmunoResearch), washed, stained with Hoechst, and mounted in VectaShield (Vector Laboratories). The images were acquired on the Zeiss LSM 710 confocal microscope. For IHC, $5 \mu \mathrm{m}$ of paraffin-embedded teratoma sections were pretreated with proteinase $\mathrm{K}$, stained with rabbit anti-Tuj1 1:200 (BioLegend), washed, and detected using a donkey anti-rabbit-HRP. DAB (3,3'diaminobenzidine) was used for visualization. Nuclei were counterstained with hematoxylin (blue).

\section{IF-FISH}

FISH probes were DIG-labeled using the DIG-Nick translation mix for in situ probes (Roche). mESCs were fixed, immunostained with mouse anti-Nup153 (1:1) and rabbit anti-LmnB2 (1:500), fixed, permeabilized in $0.1 \mathrm{M} \mathrm{HCl}$ and $0.7 \% \mathrm{TX}-100$, denatured in $50 \%$ formamide and $2 \times \mathrm{SSC}$ for $30 \mathrm{~min}$ at $80^{\circ} \mathrm{C}$, hybridized to DIG-labeled FISH probes overnight at $42^{\circ} \mathrm{C}$, stained with anti-DIG antibody (Roche), stained with Hoechst, and mounted in VectaShield (Vector Laboratories). Three-dimensional image stacks were recorded with a Zeiss LSM710 scanning scope using a $63 \times$ objective, $512 \times 512$ resolution, $2 \times$ averaging, and an optimal interval $(0.31 \mu \mathrm{m})$ between stacks in the $Z$-direction.
All BACs used in this study were purchased from the BACPAC Resource Center (BPRC) at the Children's Hospital Oakland Research Institute (CHORI) and include RP23-361N4 (Neurod1), RP23-469N2 (Nefl), RP23-129E12 (Nrp1), RP23-225O1 (Tubb3), RP23-84H23 (Ncam1), RP23-97J15 (Nlgn1), RP24-189C19 (Etv4), RP23-265D18 (En2), RP23-141A13 (Nes). RP23-281P3 (Pax6), and RP24-245P6 (Oct4/pou5f1)

\section{Antibodies}

The primary antibodies used included mouse anti-Nup153, ascites fluid (gift from B. Burke), rabbit anti-Nup153 (raised against hNup153-GST, amino acids 1300-1399), rabbit anti-Parp (9542, Cell Signaling), mouse anti-Oct-3/4 (sc-5279, Santa Cruz Biotechnology), goat anti-Oct-3/4 (sc-8628, Santa Cruz Biotechnology), mouse anti-a-tubulin (T5168, Sigma-Aldrich), mouse anti-Flag (F1804, Sigma-Aldrich), rabbit anti-Nup107 (raised against mNup107-His, amino acids 600-926), rabbit anti-Tuj1 (neuronspecific class III $\beta$-Tubulin; PRB-435P, BioLegend), mouse antiGapdh (GTX627408, Genetex), rabbit anti-Blbp (Fabp7; ab32423, Abcam), rat anti-Nestin (556309, BD pharmigen), mouse antiGata4 (sc-25310, Santa Cruz Biotechnology), rabbit anti-GFAP (Z0334, Dako), goat anti-Foxa2 (AF2400, R\&D Systems), mouse anti-a-Sma (A5228, Sigma), rabbit anti-Nup98 (2292, Cell Signaling), rabbit anti-Sox2 (2748, Cell Signaling), mouse anti-V5 (46-0705, Life Technologies), mouse anti-Ring1b (39663, Active Motif), rabbit anti-Ring1b (5694S, Cell Signaling), rabbit antiRybp (ab5976, Abcam), rabbit anti-Cbx7 (ab21873, Abcam), rabbit anti-Nup50 (ab151567, Abcam), rabbit anti-Suz12 (ab12073, Abcam), rabbit anti-Lmnb2 (ARP46356; Aviva Systems Biology), rabbit anti-Mel-18 (sc-10744, Santa Cruz Biotechnology), and rabbit anti-Foxo1 (2880S, Cell Signaling).

\section{RNA-seq, ChIP-seq, and DamID-seq}

For RNA-seq, ChIP-seq, and DamID-seq, see the Supplemental Material.

\section{Accession numbers}

The Gene Expression Omnibus accession number for the ChIPseq, RNA-seq, and DamID-seq data reported in this study is GSE64008.

\section{Acknowledgments}

We thank the members of the Hetzer laboratory for critical reading of the manuscript. We thank Dr. F. Gage and Arianna Mei for reagents and technical assistance with ESC differentiation. 16.7 mESCs were kindly provided by Dr. Jeannie Lee. We thank Dr. Bas Van Steensel for providing the pLgw EcoDam-V5-RFC1 vector. This work was supported by the National Institutes of Health grant RO1GM098749. The project described was supported by award number P30CA014118195 from the National Cancer Institute.

\section{References}

Bastos R, Lin A, Enarson M, Burke B. 1996. Targeting and function in mRNA export of nuclear pore complex protein Nup153. J Cell Biol 134: 1141-1156.

Boyer LA, Plath K, Zeitlinger J, Brambrink T, Medeiros LA, Lee TI, Levine SS, Wernig M, Tajonar A, Ray MK, et al. 2006. 
Polycomb complexes repress developmental regulators in murine embryonic stem cells. Nature 441: 349-353.

Bracken AP, Dietrich N, Pasini D, Hansen KH, Helin K. 2006. Genome-wide mapping of Polycomb target genes unravels their roles in cell fate transitions. Genes Dev 20: 1123-1136.

Brown CR, Kennedy CJ, Delmar VA, Forbes DJ, Silver PA. 2008. Global histone acetylation induces functional genomic reorganization at mammalian nuclear pore complexes. Genes Dev 22: 627-639.

Buchwalter AL, Liang Y, Hetzer MW. 2014. Nup50 is required for cell differentiation and exhibits transcription-dependent dynamics. Mol Biol Cell 25: 2472-2484.

Capelson M, Liang Y, Schulte R, Mair W, Wagner U, Hetzer MW. 2010. Chromatin-bound nuclear pore components regulate gene expression in higher eukaryotes. Cell 140: 372-383.

Casolari JM, Brown CR, Komili S, West J, Hieronymus H, Silver PA. 2004. Genome-wide localization of the nuclear transport machinery couples transcriptional status and nuclear organization. Cell 117: 427-439.

D'Angelo MA, Hetzer MW. 2008. Structure, dynamics and function of nuclear pore complexes. Trends Cell Biol 18: 456-466.

D'Angelo MA, Gomez-Cavazos JS, Mei A, Lackner DH, Hetzer MW. 2012. A change in nuclear pore complex composition regulates cell differentiation. Dev Cell 22: 446-458.

Dietrich N, Lerdrup M, Landt E, Agrawal-Singh S, Bak M, Tommerup N, Rappsilber J, Södersten E, Hansen K. 2012. RESTmediated recruitment of polycomb repressor complexes in mammalian cells. PLoS Genet 8: e1002494.

Dimaano C, Ball JR, Prunuske AJ, Ullman KS. 2001. RNA association defines a functionally conserved domain in the nuclear pore protein Nup153. J Biol Chem 276: 45349-45357.

Endoh M, Endo TA, Endoh T, Fujimura Y-I, Ohara O, Toyoda T, Otte AP, Okano M, Brockdorff N, Vidal M, et al. 2008. Polycomb group proteins Ring1A/B are functionally linked to the core transcriptional regulatory circuitry to maintain ES cell identity. Development 135: 1513-1524.

Farcas AM, Blackledge NP, Sudbery I, Long HK, McGouran JF, Rose NR, Lee S, Sims D, Cerase A, Sheahan TW, et al. 2012. KDM2B links the Polycomb repressive complex 1 (PRC1) to recognition of $\mathrm{CpG}$ islands. Elife 1: e00205.

Gonzalez I, Mateos-Langerak J, Thomas A, Cheutin T, Cavalli G. 2014. Identification of regulators of the three-dimensional polycomb organization by a microscopy-based genome-wide RNAi screen. Mol Cell 54: 485-499.

Griffis ER, Craige B, Dimaano C, Ullman KS, Powers MA. 2004. Distinct functional domains within nucleoporins Nup153 and Nup98 mediate transcription-dependent mobility. Mol Biol Cell 15: 1991-2002.

Guelen L, Pagie L, Brasset E, Meuleman W, Faza MB, Talhout W, Eussen BH, de Klein A, Wessels L, de Laat W, et al. 2008. Domain organization of human chromosomes revealed by mapping of nuclear lamina interactions. Nature 453: 948-951.

He J, Shen L, Wan M, Taranova O, Wu H, Zhang Y. 2013. Kdm2b maintains murine embryonic stem cell status by recruiting PRC1 complex to CpG islands of developmental genes. Nat Cell Biol 15: 373-384.

Kalverda B, Pickersgill H, Shloma VV, Fornerod M. 2010. Nucleoporins directly stimulate expression of developmental and cell-cycle genes inside the nucleoplasm. Cell 140:360-371.

Ku M, Koche RP, Rheinbay E, Mendenhall EM, Endoh M, Mikkelsen TS, Presser A, Nusbaum C, Xie X, Chi AS, et al. 2008. Genomewide analysis of PRC1 and PRC2 occupancy identifies two classes of bivalent domains. PLoS Genet 4: e1000242.
Leeb M, Wutz A. 2007. Ring1B is crucial for the regulation of developmental control genes and PRC1 proteins but not $\mathrm{X}$ inactivation in embryonic cells. J Cell Biol 178: 219-229.

Leeb M, Pasini D, Novatchkova M, Jaritz M, Helin K, Wutz A. 2010. Polycomb complexes act redundantly to repress genomic repeats and genes. Genes Dev 24: 265-276.

Liang Y, Franks TM, Marchetto MC, Gage FH, Hetzer MW. 2013. Dynamic association of NUP98 with the human genome. PLoS Genet 9: e1003308.

Light WH, Freaney J, Sood V, Thompson A, D'Urso A, Horvath CM, Brickner JH. 2013. A conserved role for human Nup98 in altering chromatin structure and promoting epigenetic transcriptional memory. PLOS Biol 11: e1001524.

Lupu F, Alves A, Anderson K, Doye V, Lacy E. 2008. Nuclear pore composition regulates neural stem/progenitor cell differentiation in the mouse embryo. Dev Cell 14: 831-842.

Mackay DR, Elgort SW, Ullman KS. 2009. The nucleoporin Nup153 has separable roles in both early mitotic progression and the resolution of mitosis. Mol Biol Cell 20: 1652-1660.

Makise M, Mackay DR, Elgort S, Shankaran SS, Adam SA, Ullman KS. 2012. The Nup153-Nup50 protein interface and its role in nuclear import. J Biol Chem 287: 38515-38522.

Matsuura Y, Stewart M. 2005. Nup50/Npap60 function in nuclear protein import complex disassembly and importin recycling. EMBO I 24: 3681-3689.

Mendjan S, Taipale M, Kind J, Holz H, Gebhardt P, Schelder M, Vermeulen M, Buscaino A, Duncan K, Mueller J, et al. 2006. Nuclear pore components are involved in the transcriptional regulation of dosage compensation in Drosophila. Mol Cell 21: 811-823.

Morey L, Pascual G, Cozzuto L, Roma G, Wutz A, Benitah SA, Di Croce L. 2012. Nonoverlapping functions of the Polycomb group Cbx family of proteins in embryonic stem cells. Cell Stem Cell 10: 47-62.

Pascual-Garcia P, Capelson M. 2014. Nuclear pores as versatile platforms for gene regulation. Curr Opin Genet Dev 25: 110-117.

Rabut G, Doye V, Ellenberg J. 2004. Mapping the dynamic organization of the nuclear pore complex inside single living cells. Nat Cell Biol 6: 1114-1121.

Sabri N, Roth P, Xylourgidis N, Sadeghifar F, Adler J, Samakovlis C. 2007. Distinct functions of the Drosophila Nup153 and Nup214 FG domains in nuclear protein transport. I Cell Biol 178: $557-565$.

Schmid M, Arib G, Laemmli C, Nishikawa J, Durussel T, Laemmli UK. 2006. Nup-PI: the nucleopore-promoter interaction of genes in yeast. Mol Cell 21: 379-391.

Schoeftner S, Sengupta AK, Kubicek S, Mechtler K, Spahn L, Koseki H, Jenuwein T, Wutz A. 2006. Recruitment of PRC1 function at the initiation of $\mathrm{X}$ inactivation independent of PRC2 and silencing. EMBO J 25: 3110-3122.

Shah S, Tugendreich S, Forbes D. 1998. Major binding sites for the nuclear import receptor are the internal nucleoporin Nup153 and the adjacent nuclear filament protein Tpr. I Cell Biol 141: 31-49.

Simon JA, Kingston RE. 2013. Occupying chromatin: Polycomb mechanisms for getting to genomic targets, stopping transcriptional traffic, and staying put. Mol Cell 49: 808-824.

Tavares L, Dimitrova E, Oxley D, Webster J, Poot R, Demmers J, Bezstarosti K, Taylor S, Ura H, Koide H, et al. 2012. RYBPPRC1 complexes mediate H2A ubiquitylation at polycomb target sites independently of PRC2 and H3K27me3. Cell 148: 664-678.

Toyama BH, Savas JN, Park SK, Harris MS, Ingolia NT, Yates JR, Hetzer MW. 2013. Identification of long-lived proteins reveals 
exceptional stability of essential cellular structures. Cell 154: 971-982.

Ullman KS, Shah S, Powers MA, Forbes DJ. 1999. The nucleoporin nup153 plays a critical role in multiple types of nuclear export. Mol Biol Cell 10: 649-664.

Van de Vosse DW, Wan Y, Wozniak RW, Aitchison JD. 2011. Role of the nuclear envelope in genome organization and gene expression. Wiley Interdiscip Rev Syst Biol Med 3: 147-166.

Van de Vosse DW, Wan Y, Lapetina DL, Chen W-M, Chiang J-H, Aitchison JD, Wozniak RW. 2013. A role for the nucleoporin Nup170p in chromatin structure and gene silencing. Cell 152: 969-983.

van der Stoop P, Boutsma EA, Hulsman D, Noback S, Heimerikx M, Kerkhoven RM, Voncken JW, Wessels LFA, van Lohuizen M. 2008. Ubiquitin E3 ligase Ring1b/Rnf2 of polycomb repressive complex 1 contributes to stable maintenance of mouse embryonic stem cells. PLoS One 3: e2235.

van Steensel B, Henikoff S. 2000. Identification of in vivo DNA targets of chromatin proteins using tethered dam methyltransferase. Nat Biotechnol 18: 424-428.

Vaquerizas JM, Suyama R, Kind J, Miura K, Luscombe NM, Akhtar A. 2010. Nuclear pore proteins nup153 and megator define transcriptionally active regions in the Drosophila genome. PLoS Genet 6: e1000846.
Walther TC, Fornerod M, Pickersgill H, Goldberg M, Allen TD, Mattaj IW. 2001. The nucleoporin Nup153 is required for nuclear pore basket formation, nuclear pore complex anchoring and import of a subset of nuclear proteins. $E M B O J \mathbf{2 0}$ 5703-5714.

Walther TC, Alves A, Pickersgill H, Loïodice I, Hetzer M, Galy V, Hülsmann BB, Köcher T, Wilm M, Allen T, et al. 2003. The conserved Nup107-160 complex is critical for nuclear pore complex assembly. Cell 113: 195-206.

Wente SR, Rout MP. 2010. The nuclear pore complex and nuclear transport. Cold Spring Harb Perspect Biol 2: a000562a000562.

Wu X, Johansen JV, Helin K. 2013. Fbxl10/Kdm2b recruits polycomb repressive complex 1 to $\mathrm{CpG}$ islands and regulates H2A ubiquitylation. Mol Cell 49: 1134-1146.

Yu M, Mazor T, Huang H, Huang H-T, Kathrein KL, Woo AJ, Chouinard CR, Labadorf A, Akie TE, Moran TB, et al. 2012. Direct recruitment of polycomb repressive complex 1 to chromatin by core binding transcription factors. Mol Cell 45: 330-343.

Zhang X, Chen S, Yoo S, Chakrabarti S, Zhang T, Ke T, Oberti C, Yong SL, Fang F, Li L, et al. 2008. Mutation in nuclear pore component NUP155 leads to atrial fibrillation and early sudden cardiac death. Cell 135: 1017-1027. 


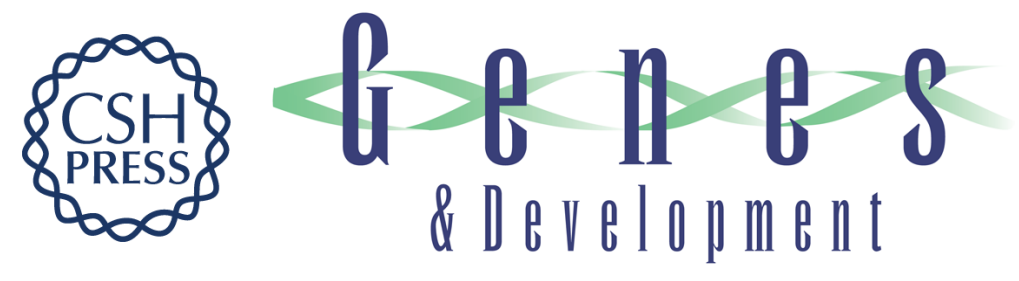

\section{The nucleoporin Nup153 regulates embryonic stem cell pluripotency through gene silencing}

Filipe V. Jacinto, Chris Benner and Martin W. Hetzer

Genes Dev. 2015, 29: originally published online June 16, 2015

Access the most recent version at doi:10.1101/gad.260919.115

\section{Supplemental http://genesdev.cshlp.org/content/suppl/2015/06/12/gad.260919.115.DC1 Material}

References This article cites 50 articles, 18 of which can be accessed free at: http://genesdev.cshlp.org/content/29/12/1224.full.html\#ref-list-1

Creative This article is distributed exclusively by Cold Spring Harbor Laboratory Press for the first Commons six months after the full-issue publication date (see

License http://genesdev.cshlp.org/site/misc/terms.xhtml). After six months, it is available under a Creative Commons License (Attribution-NonCommercial 4.0 International), as described at http://creativecommons.org/licenses/by-nc/4.0/.

Email Alerting Receive free email alerts when new articles cite this article - sign up in the box at the top Service right corner of the article or click here.

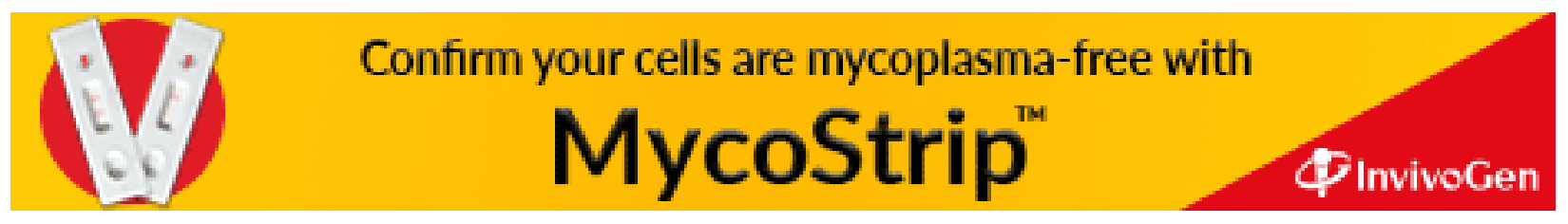

